\title{
Controlling side-effects in maintenance
}

\author{
G. Canfora* $\quad$ G. A. Di Lucca ${ }^{* *} \quad$ M. Tortorella** \\ *Dept. of 'Ingegneria dell'Informazione ed Ingegneria Elettrica' \\ University of Salerno, Faculty of Engineering at Benevento \\ ${ }^{* *}$ Dept. of 'Informatica e Sistemistica' - University of Naples 'Federico II' \\ via Claudio, 21 - 80125 Napoli, Italy \\ Tel.: +39817683199 Fax: +39817683186 \\ E-mail: canfora/dilucca/martor@nadis.dis.unina.it
}

\begin{abstract}
Each time a maintenance operation is performed on a program the overall quality of a software system can rapidly decrease because of the introduction of side effects. Such side effects are due to the relationships existing between the components of a software system, and these can be classified into potential relationships and actual relationships. Successful maintenance requires knowledge of both actual and potential relationships in order to avoid, or at least reduce to a minimum, the introduction of side-effects.

A method is proposed to analyze both potential and actual relationships existing in programs coded by a language with strict scope and visibility rules. The method is based on the definition, use and computation of some Boolean matrices and allows the identification of the software units that will be affected by a modification involving data references in a software unit.
\end{abstract}

Keywords

Software Engineering, Software Quality, Software Maintenance, Impact Analysis, Side-effects

\section{INTRODUCTION}

Software maintenance is not only one of the most expensive phases in the software life cycle (Lientz et al., 1980; Nosek et al.,1990) but it is also one of the most critical as far as quality is concerned since a bad maintenance phase can rapidly decrease the overall quality of a software system. One of the reasons why maintenance is so critical is that changes made at any one point in a system may have side effects at other points (Schneidewind, 1987). Therefore, each time a maintenance operation (whether corrective, adaptive, or perfective) has to be performed, the introduction of side effects is a considerable hazard.

Such side-effects consist of undesirable and unforeseen (but not necessarily unforeseeable) erroneous behaviours that occur as a result of a modification, in parts of the system not directly modified and possibly 'geographically' far from the point where the modification has been made (McClure et al.,1978; Freedman et al., 1982). Side effects stem from the relationships existing between the components of a software system (software units, variables, namedconstants, control structures and data-types) and usually propagate across several software units (modules, routines, etc.). These relationships mainly derive from intra- and inter-unit controlflow, data-flow, data-dependence, and data-binding. 
The side-effects most frequently introduced in a software system, and which are also the most difficult to detect, are generated by changes to variable references in statements. Such changes may modify the data-flow, data-dependence and data-binding that actually exist or can potentially exist between software units according to the scope-rules of the coding language.

Relationships between software units can be divided into two classes: (i) potential relationships, which potentially exist between two units because, according to the language scope and visibility rules, a unit may refer to any component in the other unit; and (ii) actual relationships, which actually exist between two units because the code of one of them contains direct or indirect references to some components of the other. Obviously, actual relationships are a sub-set of potential relationships (Cimitile et al., 1990).

Any given maintenance operation affects the actual relationships and can also transform potential relationships into actual ones.

Successful maintenance requires knowledge of both actual and potential relationships. In particular, a deep knowledge, understanding and analysis of potential relationships can help to avoid, or at least to reduce to a minimum, the introduction of side-effects.

Potential and actual relationships have to be identified, represented and analysed before any change can be implemented in a maintenance operation. A document that represents these relationships is the main source to which the maintainer will refer to obtain the information he needs in order to evaluate the impact of a change. Static code analysis can identify the components of a software system and the actual relationships existing among them. It can also provide the information needed to identify the potential relationships.

In this paper we propose a method to: (i) represent the potential and actual relationships between the software units in a system; (ii) analyse these relationships in order to identify automatically which other software units are affected by a change in the code of a unit.

The method is based on a matrix representation of the relationships, both potential and actual, existing between the various software units and due to the sharing of global data, the exchange of data through their interfaces and the activations of one unit by the others. The information needed to achieve this representation and the subsequent analysis is extracted from code by static analysis. This means that the data flow analysis is static and flow-insensitive, and only refers to the data references in the various software units and not also to the way in which they are arranged.

The method is described with reference to such languages as Ada, Pascal, and Modula-2, which have strict scope and visibility rules limiting the reference to components from the various units. Although these languages allow structured coding and information-hiding, they also make it more difficult to identify the potential and actual relationships.

In order to simplify the exposition, but without affecting its general nature, reference will be made to a software system made up of a single monolithic program that is in turn made up of various software units (procedure- and function-like units) rather than to a system composed of several separate software units (programs, packages, units, etc.).

\section{BACKGROUND AND MOTIVATIONS}

The changes typically made in a program concern the structure of the control-flow (introduction of new control structures and modification or deletion of existing ones), the data structure, and references to variables (their definition and use).

These changes introduce logic, semantic and performance variations regarding both the lines of code that are going to be directly modified, and thus the software units containing them, and the other parts of code (and relative units) having some relationship with the modified ones.

In actual fact the impact of a modification extends to all the artefacts of a software product (Turver et al., 1994; Queille et al., 1994), but here we will only deal the impact on code.

Before making a modification, therefore, it is essential to assess, and be aware of, the impact that this might have on the code. This entails identifying all the software areas, both inside the software unit directly affected by the change and in the others which have a relationship with it, 
that will be affected by the modification in order to reduce the possibility of side effects occurring. This will also enable a better assessment of the maintenance effort needed to implement the required change.

The effects that may be introduced by a modification of the control-flow (e.g. the introduction of a new control structure, the deletion of an existing one, or the substitution of one or more existing structures with other ones) are generally limited to the inside of the software unit in which this modification is made and do not affect the relationships with the other software units. Whereas it is much more difficult to identify areas of code affected by the modification of references to variables, for instance the modification of an expression defining the value of a variable with the addition or deletion of a reference to a variable. This type of modification may involve more than one software unit and in particular all the units that, according to the language rules, reference or can reference the variables affected by the modification and all the other variables that have a dependence relationship with them.

The impact on the code also depends on the coding language used, especially for effects due to modifications on the variable references. For instance, the impact that may arise in a program coded with a language that only allows use of global variables for all the software units of which it is made (e.g. COBOL in which the various SECTIONs and PARAGRAPHs can refer to all the variables declared in the DATA DIVISION) is different from the impact obtained with languages (such as PASCAL, ADA, PL1) that enable the declaration of variables in each of the various software units making up the program and which have rigid scope and visibility rules to discipline the possibility to reference them, according to where they have been declared. In the former case, in order to identify the regions of code that may be affected by the modification of references to variables, in an initial analysis it may be worthwhile using a cross-reference-lister which makes it possible to identify the lines of code referring to the variables involved in the modification and thus the software units affected (which may potentially be all of them). Then, use of a slicer makes it possible to further define, inside the unit, the code affected by the modification. On the contrary, in the latter case, if there is any homonymy, due to the possibility for variables declared in different units to have the same name, or any synonymity due to the exchange of actual/formal parameters in the activation of software units, this considerably reduces the utility of a cross-reference-lister. It will then be necessary to have tools that make possible a univocal identification of each variable (or other entity declared in the program), distinguishing between the various homonyms and grouping together the various synonyms. Only in this way we can be sure that more than one software unit actually references the same variable or different variables and, thus, be able to identify the units that may actually be affected by a modification in the code. Here too, intra- and inter- procedural slicing can be used to give a better definition of the areas of code affected by the modification.

Arnold (1993) defines a framework that enables comparisons to be made between the various approaches used for conducting Impact Analysis and assesses the tools currently available. One of the main characteristics that should be satisfied is that the estimation of the areas, i.e. of the software units, affected by a modification must be as close as possible to the actual one. This degree of accuracy should be reached as soon as possible so as to enable an early estimation of the modification's complexity and the resources to be dedicated to it and also to identify regions in which side effects might occur and thus reduce the possibility of their occurring.

In literature there are a number of contributions on Impact Analysis, most of which aim to establish measurements of Program Stability (Yau and Collofello, 1980), i.e. 'the resistance of a program to the amplification of changes in the program'. Yau and Collofello (1980) define metrics for assessing the resistance of a program's software units to a logic change. The metric is based on a complexity metric and on the probability that a change in a unit might affect a given variable. Yau and Chang (1984) describe a technique that is similar but more straightforward to apply for large systems. Haney (1972) presents a technique for modelling the stability of large systems based on the use of a matrix which reports the probability with which a modification in one unit will entail modifications in others. Yau and Collofello (1985) describe a stability measurement based on the counting of assumptions made on the software unit interfaces and the global data structures. 
However, these techniques are not always able to satisfy the requisite of identifying, as quickly and accurately as possible, the set of software units affected by modifications regarding variations to the variable references. To achieve this, we must first define and identify the potential and actual relationships existing between these units as a result of the type of implementation made, and thus also according to the coding language used.

\section{REPRESENTING POTENTIAL AND ACTUAL RELATIONSHIPS}

The relationships existing between the various program units making up a software system are of two fundamental types: (i) those defined by the Inter-Modular Data-flow (IMD), produced by the set of links established between pairs of units that respectively define and use the same data; (ii) those defined by the Inter-Modular Relationship (IMR), i.e. by the set of links established between pairs of units when one of the two activates the other. These relationships must be known and fully understood in order to control the effects deriving from their modification in a maintenance operation. However, these relationships are not always adequately identified by classic high level design documents, such as Structure Charts and/or Data Flow Diagrams, mainly because they are highly dependent on the programming language adopted and on the way in which the programmer has integrated the various software units according to the rules made available by the adopted language.

With reference to the two categories of actual and potential relationships and to the IMD and IMR, we can distinguish between:

- Potential Data-Flow (PDF): the set of links due to the possibility to define and use data in different program units. These links are not necessarily actually implemented in the code but knowledge of them is nevertheless important as they can be produced directly or as side effects in subsequent maintenance interventions.

- Actual Data-Flow (ADF): the set of links actually implemented in the code through the definition and use of data in different program units. We are interested in these links both for the exact identification of the effects deriving from their changes and for the side effects due to variations that do not involve them directly.

- Potential Modules Relationship (PMR): the set of links due to the possible activation of program units by other program units. These links are not necessarily actually implemented in the code but can be achieved subsequently and can produce new sequences of program unit execution directly or as side effects.

- Actual Modules Relationship (AMR): the set of links actually implemented in the code through the activation of program units by other program units. We are interested in these links both for the analysis of the consequences of variations in such links and for the side effects that their presence might produce following variations that do not involve them directly.

\subsection{Potential Relationships}

The PMR existing between two program units is generally defined by the declarative section of the program. In particular, with reference to the languages with strict scope/visibility rules it is defined by the declarative nesting of the software units of which it is made and by the visibility rules. The declarative nesting can be represented with a tree whose root is the main program; every remaining node ' $n$ ' is associated to a software unit, and an edge from $n_{i}$ to $n_{j}$ indicates that the unit $n_{j}$ is declared in the declarative section of $n_{j}$. This tree, called MDT (Module Declaration Tree), can be represented through a squared Boolean matrix MDT_mat of order $\mathrm{n}$, where $\mathrm{n}$ is the number of software units making up the program.

Rows and columns are associated to the program units; the order of association coincides with the lexicographical order with which the declarations follow each other in the source text (and thus the first row and first column will correspond to the main program, the second to the first unit declared in it, and so forth). 
If we use MDT_mat(i,j) to indicate the generic element of this matrix, we will have: - MDT_mat $(i, j)=1$ if the unit $i$ contains the declaration of the unit $j$;

- MDT_mat $(i, j)=0$ if the unit $i$ does not contain the declaration of the unit $j$.

Also the PDF depends on the declarative structure of the program: it depends on the declarative nesting of the various software units, on the data declarations made in each of these units and by the language's scope/visibility rules.

The structure of the data declarations made in the various software units can be represented by a data declarations matrix DD_mat: a Boolean $(\mathrm{n} \times \mathrm{m}$ ) matrix, where $\mathrm{n}$ is the number of units making up the program and $m$ is the number of variables with different names declared in it.

Rows and columns are associated to program units and variables, respectively; the order of association coincides with the lexicographical order with which the declarations follow each other in the source text. In the event of homonymy, i.e. declarations (in different units) of different variables with the same name, the variables are made to correspond to the same column (the one for the first declaration). In our discussion, homonyms will be univocally identified by a dot notation (unit_name.variable_name).

If we use DD_mat $(\mathrm{i}, \mathrm{j})$ to indicate the generic element of this matrix, we have:

- DD_mat $(\mathrm{i}, \mathrm{j})=0$ if the unit $\mathrm{i}$ does not contain the declaration of the variable $\mathrm{j}$;

- DD_mat $(i, j)=1$ if the unit $i$ contains the declaration of the variable $j$.

Therefore, row $\mathrm{i}$ will indicate all the variables (including the formal parameters) declared in unit $\mathrm{i}$ while column $\mathrm{j}$ will indicate in which units a variable assigned to it is declared.

\subsection{Actual Relationships}

The AMR is made up of the activations actually implemented in the code. It can be represented by a tree whose root is the main program. Every remaining node $\mathrm{n}$ is associated to a software unit, and an edge from $n_{i}$ to $n_{j}$ indicates that the unit $n_{j}$ is activated at least once by the unit $n_{i}$. This tree, called MCT (Module Call Tree), can be represented through a squared Boolean matrix MCT_mat of order $n$, having the same structure and composition as MDT_mat.

If we use MCT_mat $(\mathrm{i}, \mathrm{j})$ to indicate the generic element of this matrix, we have:

- MCT_mat $(i, j)=1$ if the unit $i$ activates the unit $j$;

- MCT_mat $(i, j)=0$ if the unit $i$ does not activate the unit $j$.

The ADF is due to the actual data references made in the various software units. These references can be represented through the Data Reference Matrix, DREF_mat, a Boolean matrix $(\mathrm{n} \times \mathrm{m})$ with the same structure and composition as DD_mat.

If we use DREF_mat(i,j) to indicate the generic element of this matrix, we have:

- DREF_mat $(\mathrm{i}, \mathrm{j})=0$ if the unit $\mathrm{i}$ does not reference the variable $\mathrm{j}$;

- DREF_mat $(i, j)=1$ if the variable $j$ is referenced in the unit $i$.

Row $i$ therefore indicates all the variables referenced by the unit corresponding to it; similarly, column $\mathrm{j}$ will indicate in which units the variable associated to it is referenced.

With reference to the Pascal-like program scheme of Figure 1, the relative MDT and MCT are illustrated in Figure 2 and the MDT_mat, MCT_mat in Figure 3, while DD_mat and DREF_mat matrices are illustrated in Figure 4.

\section{THE REPRESENTATION OF POTENTIAL AND ACTUAL RELATIONSHIPS DUE TO DATA REFERENCES}

Information on the Potential and Actual Data-Flow is important as we have to know which of the already existing links are modified or which of the possible new links are created through a maintenance operation involving the reference to a variable. In the following we show how this information can be represented using the matrix notation. 


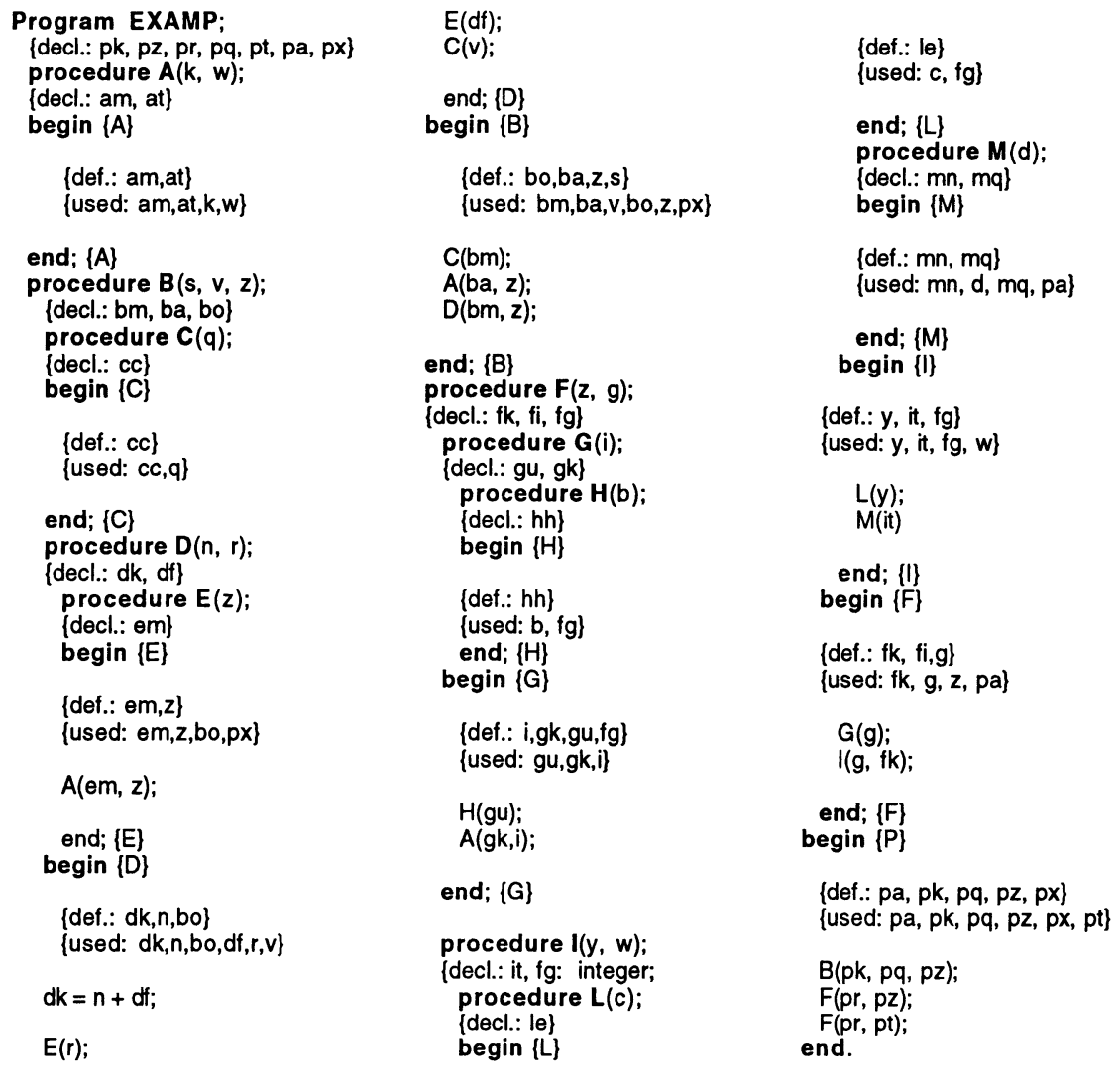

Figure 1 The scheme of the program EXAMP.

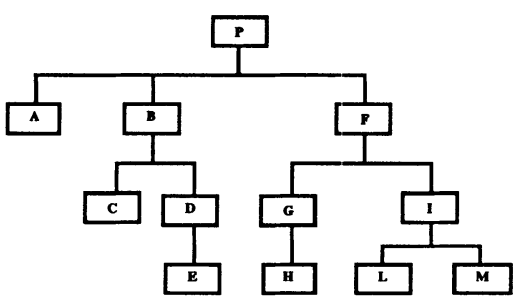

(a) The Module Declaration Tree - MDT

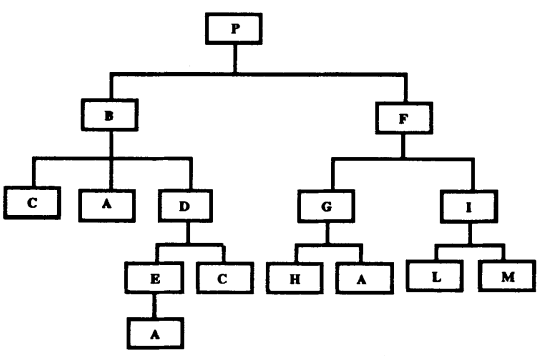

(b) The Module Call Tree - MCT

Figure 2 The MDT and MC'T of the program EXAMP. 
The scope/visibility matrix, VIS_mat

This is a Boolean matrix ( $\mathrm{n} \times \mathrm{n})$ with the same structure and composition as the MDT_mat matrix, from which it can be obtained. The matrix has the peculiarity that VIS_mat $(i, j)=1$ if, and only if, on the basis of the programming language's scope/visibility rules, the unit corresponding to row $\mathrm{i}$ can reference the variables declared in the unit corresponding to column j. VIS_mat can be determined as follows:

$$
\text { VIS_mat }(i, j)=\left((M D T+I)^{T}\right)^{+}
$$

where $I$ is the unity diagonal matrix and $\mathrm{A}^{\mathrm{T}}$ and $\mathrm{A}^{+}$indicate the transpose and the transitive closure of matrix A respectively.

Figure 3 shows the VIS_mat matrix for the program in Figure 1. Figure 3 also points out that the VIS_mat matrix shows the existence of subtrees identifying the declaration scope in each unit, and the units belonging to each subtree: these are identified by the sequences of values 1 along the columns of the VIS_mat matrix and the root of each of them corresponds to the first row of the chain containing the value 1 .

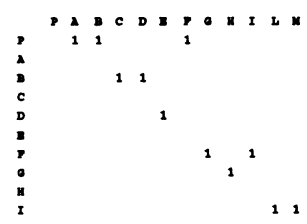

(a) MDT_mat

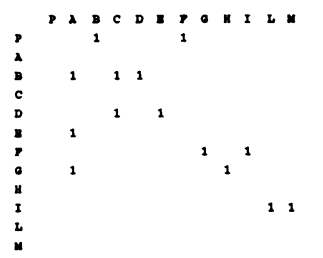

(b) MCT_mat

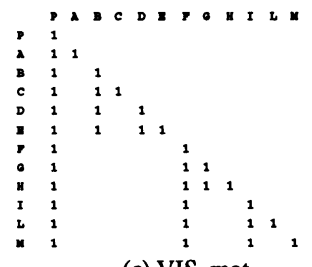

(c) VIS_mat

Figure 3 The matrices MDT_mat, MCT_mat and VIS_mat of the program EXAMP.

\section{The potential data reference matrix, $P D R \_$mat}

PDR_mat is a Boolean matrix ( $\mathrm{n} \mathrm{x} \mathrm{m}$ ) with the same structure and composition as the matrix DD_mat. PDR_mat can be obtained from the following matricial product:

$$
\text { PDR_mat }=\text { VIS_mat } x \text { DD_mat }
$$

If we use PDR_mat $(i, j)$ to indicate the generic element of this matrix, we have:

PDR_mat $(i, j)=1$ if, and only if, the unit $i$ can reference the variable corresponding to column $j$ (i.e. the declaration of variable $j$ is visible to unit $i$ ).

If the variable $\mathrm{j}$ has been declared just once in a unit ( $\mathrm{i}-\mathrm{k}$ ) which is visible to unit $\mathrm{i}$, unit $\mathrm{i}$ can reference it. Otherwise, if the variable $j$ has been declared more than once, unit $i$ can reference the last declaration, i.e. the declaration performed in the closest unit (i-k) that lexicographically precedes unit $i$ and is visible to it.

Let $x_{j}$ be a variable declared one or more times and $P_{i}$ be a unit that can reference it, i.e. PDR_mat $(i, j)=1$ where $i$ is the row corresponding to the unit $P_{i}$ and $j$ the column corresponding to the variable $x_{j}$. The variable $x_{j}$ which $P_{i}$ is referencing to is the one declared in a unit $P_{i-k}$ (corresponding to the row $\mathrm{i}-\mathrm{k}$ ) for which the following is true:

$$
\text { DD_mat }\left.\left(\mathrm{P}_{\mathrm{i}-\mathrm{k}}, \mathrm{x}_{\mathrm{j}}\right)\right|_{\mathrm{k}=0, \mathrm{i}-1}=1 \text {.and. VIS_mat }\left.\left(\mathrm{P}_{\mathrm{i}}, \mathrm{P}_{\mathrm{i}-\mathrm{k}}\right)\right|_{\mathrm{k}=0, \mathrm{i}-1}=1
$$

where $\mathrm{k}$ is equal to the first value that satisfies the above rule.

Thus, the generic row $i$ of the PDR_mat matrix gives information on the variables that can be referenced by unit $i$, while the generic column $j$ indicates the units that can reference variable $j$. Figure 4 shows the PDR_mat matrix for the program in Figure 1.

The actual data flow relationships matrix, $A D F \_$mat

ADF_mat is a Boolean matrix ( $\mathrm{n} \times \mathrm{n})$, where $\mathrm{n}$ is the number of units making up the system, and having the same structure and composition as the matrix MDT_mat. 


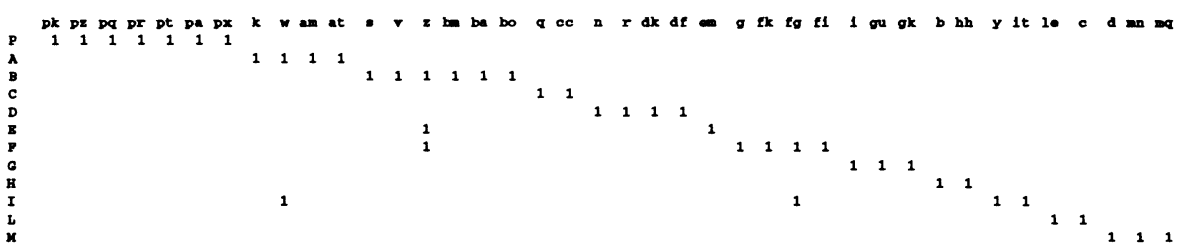

(a) DD_Mat

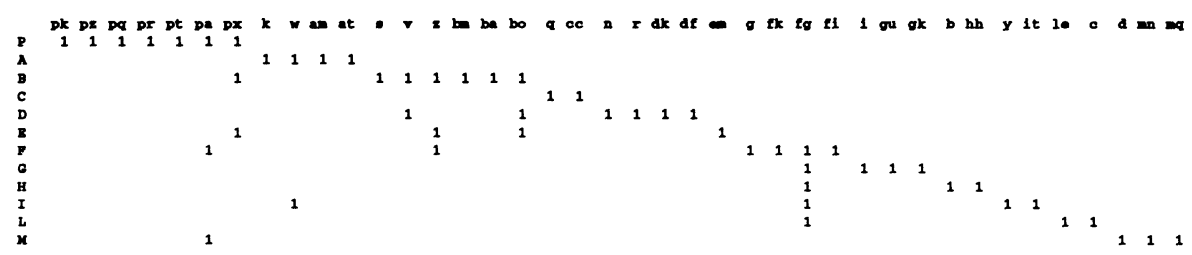

(b) DREF_Mat

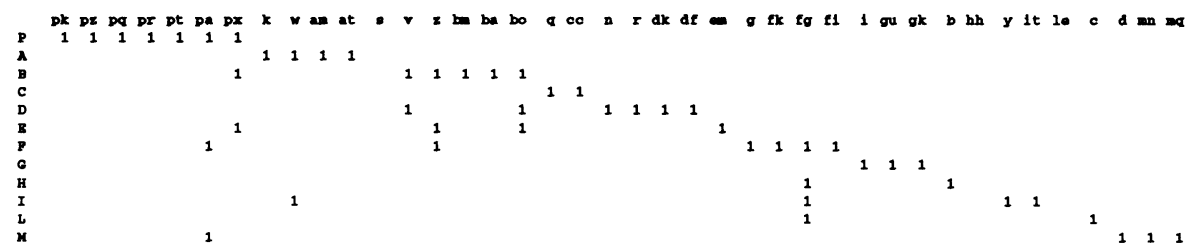

(c) DUSED_mat

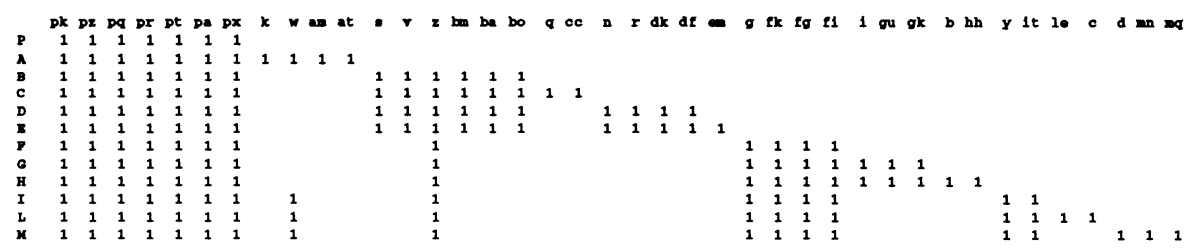

(d) PDR_mat

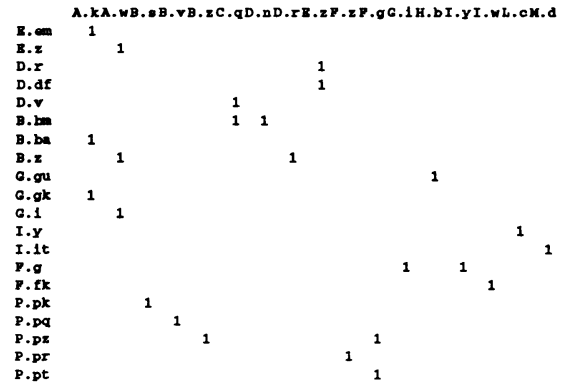

(e) PAR_ACT_FOR_mat

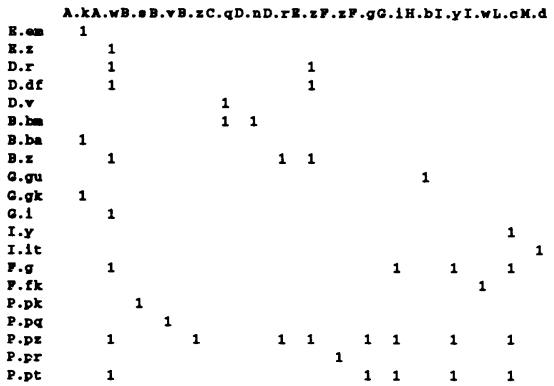

(f) PAR_ACT_FOR_mat\#

Figure 4 The matrices relative to the program EXAMP. 
We have:

ADF_mat $\left(P_{i}, P_{w}\right)=1 \Leftrightarrow \exists j \in(1, n)$ satisfying the following conditions:

1. DREF_mat $\left(\mathrm{P}_{\mathrm{i}}, \mathrm{x}_{\mathrm{j}}\right)=1$.and.DREF $\left(\mathrm{P}_{\mathrm{w}}, \mathrm{x}_{\mathrm{j}}\right)=1$;

2. DD_mat $\left.\left(\mathrm{P}_{\mathrm{i}-\mathrm{k}}, \mathrm{x}_{\mathrm{j}}\right)\right|_{\mathrm{k}=0, \mathrm{j}-1}=1$.and.VIS_mat $\left.\left(\mathrm{P}_{\mathrm{i}}, \mathrm{P}_{\mathrm{i}-\mathrm{k}}\right)\right|_{\mathrm{k}=0, \mathrm{i}-1}=1$;

3. DD_mat $\left.\left(P_{w-y}, x_{j}\right)\right|_{y=0, w-1}=1$.and.Vis_mat $\left.\left(P_{w}, P_{w-y}\right)\right|_{y=0, w-1}=1$;

4. $\mathrm{P}_{\mathrm{i}-\mathrm{k}}=\mathrm{P}_{\mathrm{w}-\mathrm{y}}$.

where $\mathrm{k}$ and $\mathrm{y}$ are equal to the first value that makes true the conditions 2 and 3 .

The conditions $1,2,3$ and 4 solve the problem caused by the homonymy between the variables.

The ADF_mat matrix is symmetrical; it represents the relationships between the various units because of the actual data flow existing between them. The generic element $A D F \_m a t ~\left(P_{i}, P_{w}\right)=1$ indicates that there is a relationship, due to the reference to the same variable, between $\mathrm{P}_{\mathrm{i}}$ and $P_{w}$. Thus the generic row $i$ (or column) of the ADF_mat matrix indicates the software units related to the unit $P_{i}$ because of their references to the same variables, and, therefore, they may be directly affected by a change made on the unit $\mathrm{P}_{\mathrm{i}}$. Figure 5 shows the ADF_mat matrix determined using the other matrices shown in the Figures 3 and 4 and the above rules.

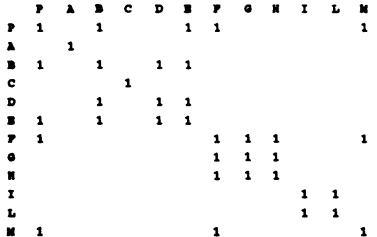

(a) ADF_mat

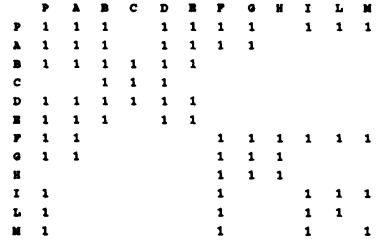

(b) ADF_mat"

Figure 5 The ADF_mat and ADF_mat ${ }^{\#}$ matrices of the program EXAMP.

\section{The actual/formal parameter matrix, PAR_ACT_FOR_mat}

This is a Boolean matrix ( $\mathrm{p} \times \mathrm{q}$ ) where $\mathrm{p}$ is the number of actual parameters exchanged in the various activations and $q$ is the number of formal parameters declared in the interface of the software units. Each actual/formal parameter is identified by its name and the name of the calling/called unit, i.e. if $\mathrm{p}_{\mathrm{x}}$ is an actual/formal parameter and $\mathrm{P}_{\mathrm{u}}$ a calling/called unit then we refer to it as $P_{u} \cdot p_{x}$. Each row of the matrix corresponds to an actual parameter identifier (even if an identifier is used in more than one call, only one row represents it), while each column corresponds to a formal parameter identifier.

For the generic element PAR_ACT_FOR_mat(i,j), we have:

- PAR_ACT_FOR_mat $(i, j)=0$ if the actual parameter $P_{u} \cdot p_{i}$ is not exchanged with the formal parameter $P_{v} \cdot P_{j}$;

-PAR_ACT_FOR_mat $(i, j)=1$ if the actual parameter $P_{u} \cdot P_{i}$ is exchanged with the formal parameter $\mathrm{P}_{\mathrm{v}} \cdot \mathrm{p}_{\mathrm{j}}$.

Synonymity problems, due to the aliasing between the formal and actual parameters, make it difficult to identify the actual variables referred in the various units. This can be overcome by actualizing the data flow for the parameter exchange between the various units. This can be achieved by substituting each reference to the formal parameters with one to the respective actual parameter (Canfora et al., 1992). The use of the PAR_ACT_FOR_mat matrix helps to solve this problem as the values ' 1 ' indicate a correspondence between the actual parameter corresponding to the subject row and the formal parameter related to the subject column. More difficult is the situation in which a formal parameter of a unit $P_{u}$ becomes in turn an actual parameter because it is exchanged along a sequence of activations. In this case, the same identifier appears both on the rows and on the columns of PAR_ACT_FOR_mat, i.e. if $P_{g} \cdot a_{i}$ 
and $P_{d} \cdot f_{j}$ indicate the names of the actual parameter corresponding to row $i$ and the formal parameter corresponding to column $j$ respectively, it may happen that $P_{g} \cdot a_{i}=P_{d} \cdot f_{j}$. To know which is the actual parameter corresponding to the formal parameter to whom is passed, as the actual one, a formal parameter of another unit, we use the following matrix PAR_ACT_FOR_mat ${ }^{\#}$, a Boolean matrix having the same structure and composition of PAR_ACT_FOR_mat; we have:

1. PAR_ACT_FOR_mat $(i, j)=1 \Rightarrow P A R \_A C T \_F O R \_m a t^{\#}(i, j)=1$;

2. $\forall \mathrm{i}^{+} \in(\overline{1}, \mathrm{p}), \overline{\mathrm{j}}^{+} \in(1, \mathrm{q}): \mathrm{P}_{\mathrm{g}} \cdot \mathrm{a}_{\mathrm{j}^{+}}=\mathrm{P}_{\mathrm{d}} \cdot \mathrm{f}_{\mathrm{j}^{+}}$find the values $\mathrm{j}^{*}$ that satisfy PAR_ACT_FOR_mat $\left(i^{+}, j^{*}\right)=1$, indicating that the formal parameter $P_{d^{-}} f_{j^{+}}$of a unit is used as the actual one in the activation to another unit, i.e. the formal parameter $P_{d} \cdot f_{j^{+}}$is passed to the ones corresponding to the columns $\mathrm{j}^{*}$ as the actual parameter $\mathrm{P}_{\mathrm{g}} \cdot \mathrm{a}_{\mathrm{i}^{+}}$;

3. $\forall \mathrm{j}^{*}$ set PAR_ACT_FOR_mat $\left.{ }^{\#}\left(\mathrm{i}, \mathrm{j}^{*}\right)\right|_{\mathrm{i}=1, \mathrm{p}}=1 \Longleftrightarrow$ PAR_ACT_FOR_mat $\left.{ }^{\#}\left(\mathrm{i}, \mathrm{j}^{+}\right)\right|_{\mathrm{i}=1, \mathrm{p}}=1$

The last operation reports the values of the column $P_{\mathrm{d}} \cdot \mathrm{f}_{\mathrm{j}^{+}}$in the column $\mathrm{P}_{\mathrm{h}} \mathrm{f}_{\mathrm{j}^{*}}$, thus establishing a direct correspondence between the actual parameter $P_{k} \cdot a_{k}$, passed to $P_{d} \cdot f_{j^{+}}$, and the parameter $\mathrm{P}_{\mathrm{h}} \mathrm{f}_{\mathrm{j} *}$.

In such a way we can know which are the actual variables that a unit refers to when it is referencing to a formal parameter.

The synonymity problems, due to the aliasing between actual and formal parameters, are solved referring to the PAR_ACT_FOR_mat".

From the matrix PAR_ACT_FOR_mat ${ }^{\#}$ we can compute the matrix ADF_mat ${ }^{\#}$, which is a Boolean matrix ( $\mathrm{n} \times \mathrm{n}$ ) having the same structure and composition of ADF_mat. In ADF_mat we have the same kind of inforrnation that is in ADF_mat but it also gives us information about the relationships among the units due to the aliasing of formal parameter.

Let $\mathrm{P}_{\mathrm{i}}$ and $\mathrm{P}_{\mathrm{j}}$ be the units corresponding to row $\mathrm{i}$ and column $\mathrm{j}$ respectively, we can compute ADF_mat ${ }^{\#}$ by applying the following rules :

1. $A D F \_m a t ~(i, j)=1 \Rightarrow A D F \_m a t^{\#}(i, j)=1$;

2. ADF_mat ${ }^{\#}(i, j)=1 \Longleftrightarrow \exists(k, q):$ PAR_ACT_FOR_mat ${ }^{\#}(k, q)=1$.and. $P_{i}$ is the unit whose actual parameter, corresponding to the row $k$ of the last matrix, reaches the formal parameter, corresponding to the column $q$, of the unit $P_{j}$ along a sequence of activations.

Figure 4 shows the PAR_A.CT_FOR_mat and PAR_ACT_FOR_mat" matrices for the program in Figure 1, while in Figure 5 is the ADF_mat" matrix for the same program.

\section{THE IDENTIFICATION OF THE SOFTWARE UNITS AFFECTED BY A CHANGE}

We use the matrices defined in the previous sections to identify the software units that may be affected by a change to be made in a unit $P_{\mathrm{i}}$. Let us denote with:

- $P_{i}$, the software unit to be modified in order to make a change;

- MV, the set of the variables $v_{j}$ involved by the change. We can get the MV set by:

where:

$$
\mathrm{MV}=\mathrm{IV} \cup \mathrm{DV} \cup \mathrm{AV} \cup \mathrm{FV}
$$

- IV is the set of the variables directly involved in the change, i.e. the ones referred to in the statement to be changed:

IV $=\left\{v_{i}\right.$ : reference to $v_{i}$ in $P_{i}$ to be modified $\}$.

- DV is the set of the variables having a dependence relationships, inside the unit $P_{i}$, with the ones in the set IV.

- $\mathrm{AV}$ is the set of actual parameter corresponding to any formal one of $\mathrm{P}_{\mathrm{i}}$ being in IV $\cup \mathrm{DV}$ : 
$A V=\left\{v_{a}: \exists v_{i} \in(I V \cup D V), v a \in A P:\left.P A R \_A C T \_F O R \_m a{ }^{\#}\left(P_{a} \cdot v_{a}, P_{i} \cdot v_{i}\right)\right|_{a=1, p}=1\right\}$ where AP is the set of actual parameters, corresponding to the rows of the matrix PAR_ACT_FOR_mat.

- FV is the set of formal parameters to which any variable in IV $\cup D V$ is passed:

$$
F V=\left\{v_{f}: \exists v_{i} \in(I V \cup D V), v_{f} \in F P:\left.P A R \_A C T \_F O R \_m a t^{\#}\left(P_{i} \cdot v_{i}, P_{f} \cdot v_{f}\right)\right|_{f=1, q}=1\right\}
$$

where FP is the set of formal parameters, corresponding to the columns of the matrix PAR_ACT_FOR_mat.

Let P_REF_ALL be, the set of all the software units that have a relationship with $P_{i}$ because they reference the same set of variables:

$$
\text { P_REF_ALL }=\left\{P_{k}:\left.A D F \_m a t ~\left(P_{i}, P_{k}\right)\right|_{k=1, n ; k \neq i}=1\right\}
$$

we have to determine the sub-set PV $\subseteq$ P_REF_ALL made up of the software units referencing only the variables in the set MV.

The set PV will thus be made up of all the units $P_{j} \in P \_R E F \_A L L$ that make true at least one of the following conditions:

- for each variable $v_{j} \in(I V \cup D V)$ all the following conditions must be true:

1. DREF_mat $\left(\mathrm{P}_{\mathrm{i}}, \mathrm{v}_{\mathrm{j}}\right)=1$.and.DREF_mat $\left(\mathrm{P}_{\mathrm{j}}, \mathrm{v}_{\mathrm{j}}\right)=1$;

2. DD_mat $\left.\left(\mathrm{P}_{\mathrm{i}-\mathrm{k}}, \mathrm{v}_{\mathrm{j}}\right)\right|_{\mathrm{k}=0, \mathrm{i}-1}=1$.and.VIS_mat $\left.\left(\mathrm{P}_{\mathrm{i}}, \mathrm{P}_{\mathrm{i}-\mathrm{k}}\right)\right|_{\mathrm{k}=0, \mathrm{i}-1}=1$;

3. DD_mat $\left.\left(P_{j-y}, v_{j}\right)\right|_{y=0, j-1}=1$.and.VIS_mat $\left.\left(P_{j}, P_{j-y}\right)\right|_{y=0, j-1}=1$;

4. $P_{\mathrm{i}-\mathrm{k}}=\mathrm{P}_{\mathrm{j}-\mathrm{y}}$.

where $k$ and $y$ are equal to the first value making conditions 2 and 3 true.

- for each variable $v_{a} \in A V$ and $v_{j} \in(I V \cup D V)$ all the following conditions must be true:

1. DREF_mat $\left(\mathrm{P}_{\mathrm{i}}, \mathrm{v}_{\mathrm{j}}\right)=1$.and.DREF_mat $\left.\left(\mathrm{P}_{\mathrm{j}}, \mathrm{v}_{\mathrm{a}}\right)\right|_{\mathrm{j}=1, \mathrm{n}}=1$;

2. PAR_ACT_FOR_mat $\left.{ }^{\#}\left(\mathrm{P}_{\mathrm{j}} \cdot \mathrm{v}_{\mathrm{a}}, \mathrm{P}_{\mathrm{i}} \cdot \mathrm{v}_{\mathrm{j}}\right)\right|_{\mathrm{j}=1, \mathrm{p}}=1$.

- for each variable $v_{f} \in F V$ and $v_{j} \in(I V \cup D V)$ all the following conditions must be true:

1. DREF_mat $\left(\mathrm{P}_{\mathrm{i}}, \mathrm{v}_{\mathrm{j}}\right)=1$.and.DREF_mat $\left.\left(\mathrm{P}_{\mathrm{j}}, \mathrm{v}_{\mathrm{f}}\right)\right|_{\mathrm{j}=1, \mathrm{n}}=1$;

2. PAR_ACT_FOR_mat $\left.{ }^{\#}\left(P_{j} \cdot v_{j}, P_{j} \cdot v_{f}\right)\right|_{j=1, q}=1$.

The set $\mathrm{PV}$ has been computed under the hypothesis that the change to be made will not insert into $P_{i}$ new references to variables not already referenced in it before, and it will not delete any of the existing references, i.e. the change will not modify the values of the DREF_mat matrix and it will thus not create new actual relationships between the software units.

New actual relationships between software units will, however, be created if the change inserts new references to variables not referenced before. This means that in the DREF_mat matrix some values of some elements DREF_mat $\left(\mathrm{P}_{\mathrm{i}}, \mathrm{v}_{\mathrm{j}}\right)$ will change from 0 to 1 for some $\mathrm{v}_{\mathrm{j}} \in \mathrm{MV}$. We can identify the new software units affected by the change, before changing the code, by modifying the DREF_mat matrix, setting DREF_mat $\left(P_{i}, v_{j}\right)=1$ where $v_{j}$ is the new referenced variable, and computing from this the new $A D F \_$mat' matrix and the matrix ADF_mat", in which the new actual links created by that change are shown. The new actual links can be computed by: ADF_mat" - ADF_mat" .

In order to insert into a software unit $P_{i}$ a reference to a variable $v_{j}$ not previously referred in it, we must first make the following considerations:

1. check that the unit $P_{i}$ can refer: the variable $v_{j}$ and this is possible if and only if:
1.1. PDR_mat $\left(\mathrm{P}_{\mathrm{i}}, \mathrm{v}_{\mathrm{j}}\right)=1$;
1.2. DD_mat $\left.\left(\mathrm{P}_{\mathrm{i}-\mathrm{k}}, \mathrm{v}_{\mathrm{j}}\right)\right|_{\mathrm{k}=0, \mathrm{i}-1}=1$.and. VIS_mat $\left.\left(\mathrm{P}_{\mathrm{i}}, \mathrm{P}_{\mathrm{i}-\mathrm{k}}\right)\right|_{\mathrm{k}=0, \mathrm{i}-1}=1$;

that is $P_{i}$ has the visibility of the variable it has to reference.

2. check that the variable $v_{j}$ is declared in $P_{i}$ itself, or it is a global variable for $P_{i}$ :

2.1. DD_mat $\left(\mathrm{P}_{\mathrm{i}}, \mathrm{v}_{\mathrm{j}}\right)=1$;

2.2. (PDR_mat $\left.\left(\mathrm{P}_{\mathrm{i}}, \mathrm{v}_{\mathrm{j}}\right)=1\right)$.and. $\left(\mathrm{DD} \_\mathrm{mat}\left(\mathrm{P}_{\mathrm{i}}, \mathrm{v}_{\mathrm{j}}\right)=0\right)$. 
In the case of 2.1, if the unit $P_{i}$ is a leaf in the Module Declaration Tree $\left(P_{i}\right.$ does not declare any other software unit), i.e.MDT_mat $\left.(i, j)\right|_{j=1, n}=0$, the insertion of the new reference into $P_{i}$ does not create new actual links and thus ADF mat ${ }^{\# \prime}=A D F$ mat $t^{\#}$.

In the case of 2.2 or 2.1 , but where $P_{i}$ is not a leaf in the Module Declaration Tree, we have to identify the new actual links that exist between $\mathrm{P}_{\mathrm{i}}$ and the other software units referencing the same variable $v_{j}$, by analyzing the matrix ADF_mat" and computing the set PV.

We can make similar considerations if the change to be made entails the deletion of a reference to a variable $v_{\mathrm{j}}$. Here too we have to modify the DREF_mat matrix, this time setting the value of the element DREF_mat $\left(\mathrm{P}_{\mathrm{i}}, \mathrm{v}_{\mathrm{j}}\right)$ from one to zero, and then compute the ADF_mat ${ }^{\# \prime}$ matrix and evaluate the set PV.

The set PV includes all the software units referencing the same set of variables referred in $P_{i}$, but this does not mean that there is necessarily an ADF link between all the pairs $\left(P_{i}, P_{j}\right)$ : there will be an ADF link only if one of the two units references a variable $v_{i}$ to define its value, and the other unit references $v_{i}$ to use its value.

Our analysis does not allow the computation of the sequences of definitions and uses of a variable $v_{j}$ but we can only know in which software units $v_{j}$ is referenced. Thus we cannot determine when a variable is defined or used in a unit (i.e. whether it is first defined and then used or vice versa, or whether it is defined and/or used more than once, and so on) but only if it is defined and/or used and if it is only defined or only used in a unit. Therefore, we can only know which are the pairs $\left(\mathrm{P}_{\mathrm{i}}, \mathrm{P}_{\mathrm{j}}\right)$ where a variable is only defined or only used in both the units and in this case we delete the unit $P_{j}$ from the set PV. When, on the other hand, a variable $v_{j}$ is both defined and used in the units $P_{i}$ and $P_{j}$ we do not know if its use in one unit follows or precedes its definition in the other, i.e. whether we have a sequence $\operatorname{def}\left(P_{i}, v_{j}\right)-\operatorname{def}\left(P_{j}, v_{j}\right)$ use $\left(P_{j}, v_{j}\right)$ or a sequence $\operatorname{def}\left(P_{i}, v_{j}\right)-u s e\left(P_{j}, v_{j}\right)-\operatorname{def}\left(P_{j}, v_{j}\right)$. In this case we cannot know if there actually is an ADF link between the units $\left(\mathrm{P}_{\mathrm{i}}, \mathrm{P}_{\mathrm{j}}\right)$ so we assume the worst case, that is we assume that an ADF link exists between $\left(\mathrm{P}_{\mathrm{i}}, \mathrm{P}_{\mathrm{i}}\right)$ due to $\mathrm{v}_{\mathrm{i}}$.

In order to delete from the set PV the units that do not have an ADF link with $\mathrm{P}_{\mathrm{i}}$, for the above mentioned reasons, we define the following two matrices:

- the used variables matrix, DUSED_mat, a (n x m ) Boolean matrix, with the same structure and composition as the DD_mat matrix; it will be:

DUSED_mat $(i, j)=1$ if and only if in the unit corresponding to row $i$ there is a 'use' (the variable is referenced without changing its value) of the variable corresponding to column $\mathrm{j}$ at least once.

- the defined variables matrix, DDEF_mat, a $(\mathrm{n} \times \mathrm{m})$ Boolean matrix, with the same structure and composition as the DD_mat matrix; it will be:

DDEF_mat $(i, j)=1 \quad$ if and only if in the unit corresponding to row $i$ there is a 'definition' (the variable is referenced and its value is changed) of the variable corresponding to column $\mathrm{j}$ at least once.

Of course, DREF_mat $=$ DDEF_mat $\cup$ DUSED_mat.

Figure 4 shows the matrix DUSED_mat of the program in Figure 1.

We have to remove from the set $P V$ all the units $P_{j} \in P_{-}$NOT_IMP where P_NOT_IMP is the set defined as follows:

P_NOT_IMP =

$\left\{\bar{P}_{j}:\right.$ DDEF_mat $\left(P_{i}, v_{i}\right)=1$.and. (DUSED_mat $\left(P_{j}, v_{i}\right)=0$.and. $\operatorname{DDEF}$ mat $\left.\left(P_{j}, v_{i}\right)=1\right)$

or. DDEF_mat $\left(P_{j}, v_{i}\right)=1$.and. (DUSED_mat $\left(P_{i}, v_{i}\right)=0$.and. $\operatorname{DDEF}$ mat $\left.\left(P_{i}, v_{i}\right)=1\right)$

.or. DUSED_mat $\left(P_{i}, v_{i}\right)=1$ and. (DUSED_mat $\left(P_{j}, v_{i}\right)=1$.and. DDEF_mat $\left(P_{j}, v_{i}\right)=0$ )

.or. DUSED_mat $\left(\mathrm{P}_{\mathrm{j}}, \mathrm{v}_{\mathrm{i}}\right)=1$ and. (DUSED_mat $\left(\mathrm{P}_{\mathrm{i}}, \mathrm{v}_{\mathrm{i}}\right)=1$.and. $\left.\left.\operatorname{DDEF} \_\operatorname{mat}\left(\mathrm{P}_{\mathrm{i}}, \mathrm{v}_{\mathrm{i}}\right)=0\right)\right\}$

where $v_{i} \in M V$ and $P_{j} \in P V$.

The unit $P_{j}$ is in $P \_N O T \_I M P$ if the variable $v_{i}$, or any actual/formal parameter corresponding 
to it in $P_{j}$, is only defined or only used both in $P_{i}$ and $P_{j}$.

In all the other cases we consider to have an ADF link between the pairs $\left(P_{i}, P_{j}\right)$.

Finally, we can define the set P_IMP as:

$$
\text { P_IMP = PV - P_NOT_IMP }
$$

which is the set made up of the software units referencing the variables in MV and that are affected by the change to be made in $\mathrm{P}_{\mathrm{i}}$.

Once the set P_IMP has been defined, we have useful information for estimating the impact of the change to be made and the resources to dedicate to it. The estimate will depend on the number of affected units and on the complexity of each unit. Moreover we have to consider that the change may have repercussions, passing from the units in the set P_IMP to other units having a relationship with the ones in $\mathrm{P}_{\text {_IMP }}$ but not with $\mathrm{P}_{\mathrm{i}}$. Thus, for each unit in $\mathrm{P}_{-} \mathrm{IMP}$, we have to make an analysis similar to the one made above regarding $P_{i}$.

As an example, we apply the method to the program whose scheme, in a Pascal-like format, is in Figure 1.

Let us suppose to have to modify, in the unit D of the Program EXAMP, the statement

$$
\mathrm{dk}=\mathrm{n}+\mathrm{df} \text {; }
$$

by adding the variable bo, that is the changed statement will be:

$$
\mathrm{d} k=\mathrm{n}+\mathrm{df}+\mathrm{bo} \text {; }
$$

As it results by the matrices PDR_mat, DD_mat and VIS_mat, the unit D can refer to the variable bo. Thus it is possible to make the desired change. In order to estimate its impact, we have to identify which are the units affected by the change.

We have that the sets IV, DV, FV and AV are:

$$
\begin{aligned}
& I V \cup D V=\{\text { B.bo, D.dk, D.n, D.df }\} \\
& F V=\{\text { A.w, E.z }\} \\
& A V=\{\text { B.bm }\}
\end{aligned}
$$

so that the set MV will be:

$$
M V=\{B . b o, \text { D.dk, D.n, D.df, A.w, E.z, B.bm }\} .
$$

From the matrix ADF_mat ${ }^{\#}$ we obtain the set

$$
\text { P_REF_ALL }=\{P, A, B, C, E\}
$$

of the units that have a relationship with $D$ because they reference the same variables referred by D. By applying the rules we told about above, we can determine the set

$$
\mathrm{PV}=\{\mathrm{A}, \mathrm{B}, \mathrm{E}\}
$$

of the units in P_REF_ALL that reference the variables in MV, and the set

$$
\text { P_NOT_IMP }=\{\mathrm{A}\}
$$

and, finally, we can determine the set $P_{-} I M P=\{B, E\}$ that will be the units affected by that change in the unit $D$.

\section{CONCLUSIONS}

Every time we have to make a change in a program we must carefully evaluate its impact on the code by means of a thorough understanding of the software to modify in order to reduce the considerable hazard of generating side-effects. Side-effects stem from the relationships existing between the components of a software system and depend on the implementation structure and on the programming language used. The links and relationships that can exist between the several software units of a program differ according to the different syntactic, scope and visibility rules of each language. For these reasons there are a number of different reasons why a side-effect arises. Therefore, the way a program is represented is important and this representation must also take into consideration the language rules that make it possible to establish relationships between the various software units.

In the paper we have proposed a method that allows the representation of both potential and actual relationships existing in a program coded by a language with strict scope and visibility rules (such as ADA, PASCAL, MODULA-2, PL1). The proposed representation is based on 
the definition, use and computation of some Boolean matrices and it allows us to identify the software units that will be affected by a change involving data references in a software unit. The method makes it possible to identify the set of units that will be directly affected by the change, before it is actually made, and to have information to estimate the impact of the change on the code and the resources to devote to achieve it.

\section{REFERENCES}

Arnold, R. S. and Shawn, A. B. (1993) Impact Analysis - Towards a Framework for Comparison, in Proc. of the IEEE Conference on Software Maintenance (IEEE Comp. Soc. Press), Montreal, Canada, 292-301.

Canfora, G. and Cimitile, A. (1992) Reverse Engineering and Intermodular Data Flow: A Theoretical Approach. Software Maintenance: Research and Practice, 4, 37-59.

Cimitile, A., Di Lucca, G. A. and Maresca, P. (1990) Maintenance and Intermodular Dependencies in Pascal Environment, in Proc. of the IEEE Conference on Software Maintenance (IEEE Comp. Soc. Press), San Diego, California, 72-83.

Freedman, D. and Weinberg, G. (1982) A Cheklist for Potential Side Effects of a Maintenance Change, in Techniques of Program and System Maintenace, (ed. G. Parik), Winthrop Publishers.

Haney, F. M. (1972) Module Connection Analysis, in Proc. AFIPS Joint Computer Conference, 41 (5), 173-9.

Lientz, B. P. and Swanson, E. B. (1980) Software Maintenance Management. Addison Wesley.

McClure, C. (1978) Managing Software Development and Maintenance. Van Nostrand Reinhold Company, New York.

Nosek, T. J. and Palvia, P. (1990) Software Maintenance Management: Changes in the Last Decade. Journal of Software Maintenance, 2 (3), 157-174.

Queille, J. P., Voidrot, J. F., Wilde, N. and Munro, M. (1994) The Impact Analysis Task in Software Maintenance: A model and a Case Study, in Proc. of the IEEE Conference on Software Maintenance (IEEE Comp. Soc. Press), Victoria, Canada, 234-242.

Schneidewind, N. (1987) The State of Software Maintenance. IEEE Trans. on Software Engineering, SE-13, 303-310.

Turver, R. J. and Munro, M. (1994) An Early Impact Analysis Technique for Software Maintenance. Software Maintenance: Research and Practice, 6, 35-52.

Yau, S. S. and Collofello, J. S. (1980) Some Stability Measures for Software Maintenance, IEEE Trans. on Software Engineering, SE-6, (6), 545-552.

Yau, S. S. and Chang S. C. (1984) Estimating logical stability in Software Maintenance, in Proc. IEEE C.S. Computer Software and Application Conference (IEEE Comp. Soc. Press), 109-119.

Yau, S. S. and Collofello, J. S. (1985) Design Stability Measures for Software Maintenance. IEEE Trans. on Software Engineering, SE-11 (9), 849-856. 